LUNG CANCER

\title{
Prognostic significance of cyclooxygenase-2 (COX-2) and expression of cell cycle inhibitors p21 and p27 in human pleural malignant mesothelioma
}

\author{
A Baldi, D Santini, F Vasaturo, M Santini, G Vicidomini, M Pia Di Marino, V Esposito, A M Groeger, \\ G Liuzzi, B Vincenzi, G Tonini, M Piccoli, F Baldi, S Scarpa
}

Thorax 2004;59:428-433. doi: 10.1136/thx.2003.008912

See end of article for authors' affiliations

Correspondence to: Dr A Baldi, Via G Orsi 25, 80128 Naples, Italy; alfonsobaldi@tiscali.it

Received 16 April 2003 Accepted 4 February 2004
Background: A study was undertaken to analyse the potential prognostic value of the immunohistochemical expression of cyclooxygenase-2 (COX-2) and p27 in 29 malignant mesotheliomas already screened for the expression of $\mathrm{p} 21$ and $\mathrm{p} 53$.

Methods: Immunohistochemistry was used to determine the expression of COX-2 and p27. The correlation with survival of these factors and of $\mathrm{p} 21$ and $\mathrm{p} 53$ expression was assessed by univariate and multivariate analyses.

Results: A positive statistically significant correlation was found between p27 and p21 expression $(p<0.0001)$, but there was a negative correlation between COX-2 expression and both $p 27(p=0.001)$ and p21 $(p<0.0001)$. No statistically significant correlation was recorded between p53 and all the other immunohistochemical parameters. Univariate analysis showed that overall survival was strongly influenced by $\mathrm{p} 21$, p27, and COX-2 expression, but multivariate Cox regression analysis showed that the only immunohistochemical parameter to influence overall survival of patients with mesothelioma was COX-2. Conclusions: These findings suggest that COX-2 expression may be a useful prognostic parameter for mesothelioma.
M alignant mesothelioma is a rare, highly aggressive tumour which accounts for less than $1 \%$ of all cancer deaths in the world. ${ }^{1}$ This neoplasm arises from the surface serosal cells of the pleural ( $>90 \%$ of cases), peritoneal, and pericardial cavities and from the tunica vaginalis of the testis. ${ }^{2}$ Although the association between exposure to asbestos and the development of mesothelioma is commonly accepted, the exact mechanism whereby asbestos induces the mesothelioma is unknown. ${ }^{3}$ Several studies report the presence in some mesotheliomas of DNA encoding SV40 $\mathrm{T}$ antigen or SV40 $\mathrm{T}$ antigen protein expression, suggesting that the presence of this viral gene may also be associated with the pathogenesis of this neoplasm. ${ }^{4}$ The prognosis is generally poor with a reported median survival of 4-12 months in either untreated or treated (surgery, radiotherapy, or chemotherapy) patients. ${ }^{5}$ Moreover, mesothelioma has proved resistant to classical chemotherapeutic and radiation regimes and the natural history has not been influenced by standard therapy. ${ }^{6}$ Several clinical prognostic factors have been tentatively correlated to survival, histological type and performance status being the most valuable. ${ }^{5}$ 7-9

Cyclooxygenase-2 (COX-2) has been implicated in carcinogenesis of several neoplasms through the downregulation of cell mediated immunity, promotion of angiogenesis, inhibition of apoptosis, and the formation of carcinogenic metabolites such as malondialdehyde. ${ }^{10-12}$ Furthermore, COX2 overexpression has been noted in many solid tumours and has been correlated with a worse prognosis in colorectal cancer, non-small cell lung cancer, and gastric cancer. ${ }^{13-15}$ It has been shown that cultured human mesothelial cells contain cyclooxygenase activity ${ }^{16}$ and that COX-2 expressing mesothelioma cell lines are associated with increased proliferative and invasive potential. ${ }^{17} \mathrm{~A}$ recent study showed that COX-2 expression is a strong prognostic factor in human mesothelioma, which contributes independently to the other clinical and histopathological factors in determining a short survival. ${ }^{18}$ It has been proposed that COX-2 exerts its influence on mesangial cell proliferation in vitro by a novel mechanism involving the tumour suppressor p53 and the cell cycle inhibitors p 21 and p27, ${ }^{19}$ and several recent studies have investigated the potential prognostic value of p53, p21 and p27 in malignant mesotheliomas. ${ }^{20-24}$

A study was undertaken to analyse the potential prognostic value of the immunohistochemical expression of COX-2 and p27 in 29 malignant mesotheliomas already screened for the expression of $\mathrm{p} 21$ and $\mathrm{p} 53^{23} 24$ and for the presence of SV40like sequences. ${ }^{25}$ The correlation with survival of each factor in univariate and multivariate analysis was assessed.

\section{METHODS}

Clinical data and tumour specimen acquisition

All patients were treated at the Second University of Naples between 1980 and 1996. Clinical data were obtained by retrospective chart review. Survival was determined from the date of initial surgery. Follow up was available for all patients. No patients were excluded from survival analysis because of death unrelated to cancer, and no patients were lost during follow up. The two patients not included in the analysis of survival were excluded because they died in the postoperative period from surgery related complications.

All patients underwent cytoreductive surgery and 13 patients were further treated with radiotherapy or chemotherapy. Only in three cases did the clinical history show a clear exposure to asbestos, but this does not mean that the other patients were not also exposed to asbestos. In fact, all the patients were living in Campania, a region where contamination with asbestos is very high. Moreover, cases were selected who were still available for immunohistochemical studies from the original group of 35 already characterised for the presence of SV40 sequences. ${ }^{25}$ 
Tissue from 29 malignant mesothelioma specimens (16 epithelioid, six sarcomatoid, and seven mixed mesotheliomas) obtained from open biopsies or pleurectomies were collected and fixed in $10 \%$ formalin before being embedded in paraffin.

\section{Histological examination}

Five $\mu \mathrm{m}$ sections were cut from the formalin fixed, paraffin embedded samples and stained with haematoxylin and eosin. The histological diagnosis was re-examined by two pathologists ( $\mathrm{AB}$ and $\mathrm{FB}$ ) according to the World Health Organization histological typing of tumours. In addition, the most representative blocks were selected to be cut into new $5 \mu \mathrm{m}$ thick sections for immunohistochemical studies.

\section{Immunohistochemistry}

All 29 cases had been assessed by immunohistochemistry for the presence of COX-2 and p27. Five $\mu \mathrm{m}$ sections were cut from each specimen, mounted on glass, and dried overnight at $37^{\circ} \mathrm{C}$. All sections were then deparaffinised in xylene, rehydrated through a graded alcohol series, and washed in phosphate buffered saline (PBS). PBS was used for all subsequent washes and for antibody dilution. Endogenous peroxidase activity was blocked by $5 \%$ hydrogen peroxide. The primary antibodies for p27 (mouse monoclonal; sc-1641, Santa Cruz Biotechnology, CA, USA) and for COX-2 (goat polyclonal; sc-1745, Santa Cruz Biotechnology) were applied at room temperature for 1 hour at a dilution of 1:100. The optimal working dilution was defined on the basis of titration experiments. The sections were then immunostained with the streptavidin-biotin system (Dako, Carpintera, CA, USA) using diaminobenzidine (DAB) as the final chromogen and haematoxylin as the nuclear counterstain. Negative controls for each tissue section were prepared by omitting the primary antibodies. A suitable positive control was run with each set of slides. All samples were processed under the same conditions.

The experimental conditions for the immunohistochemical analysis of p21 and p53, as well as for the detection of SV40 sequences, have been described elsewhere. ${ }^{23-25}$

\section{Scoring and quantification of immunoreactivity}

Two observers ( $\mathrm{AB}$ and SS) estimated the staining pattern of p27 and COX-2 separately. For p27, each specimen was scored for the percentage of positive nuclei $(<10 \%$ and $>10 \%$ of cells expressing p27). For COX-2, staining intensity was scored as 0 (negative), 1 (weak), 2 (medium), and 3 (strong). The extent of staining was scored as $0(0 \%), 1(1-25 \%), 2$ $(26-50 \%), 3(51-75 \%)$, and $4(76-100 \%)$ according to the percentage of positive stained cells. The sum of the intensity and extent scores was used as the final staining score (0-7). The level of concordance, expressed as the percentage of agreement between the observers, was 93\% (27 of 29 specimens). In the remaining specimens the score was obtained after collegial revision and agreement. The scoring and quantitation of immunoreactivity for p21 and p53 has been described elsewhere. ${ }^{23} 24$

To select the cut off point for COX-2 expression the samples were divided into two groups ( $0-4$ and $>4)$ to give a low expression and high expression group. For all the other markers a cut off point of $10 \%$ was chosen because they are all nuclear molecules involved in cell cycle regulation whose loss of expression is strictly connected with tumour progression. A value of $10 \%$ therefore indicates whether or not the samples are expressing each of these markers.

\section{Statistical analysis}

Fisher's exact test was used to assess the relationship between ordinal data (correlation matrix between immuno- staining parameters). The correlation between COX-2, p27, and p2l was performed on the original ordinal units using the Spearman test. Univariate survival analysis for each prognostic variable on overall survival was estimated according to the Kaplan-Meier method. ${ }^{26}$ The terminal event was death attributable to cancer. The statistical significance of the differences in survival distribution between the prognostic groups was evaluated by the log-rank test. ${ }^{27}$ The Cox proportional hazards model was applied to the multivariate survival analysis. ${ }^{28}$ The prognostic variables on overall survival included histological types, COX-2, p21, p27, and p53 expression. $p$ values of $<0.05$ were regarded as statistically significant in two tailed tests. SPSS software version 10.00 (SPSS, Chicago, USA) was used for statistical analysis.

\section{RESULTS}

\section{Histology and immunohistochemistry}

The results of immunohistochemical analysis of 29 mesothelioma specimens are shown in table 1. Histologically, there were 16 epithelioid, six sarcomatoid, and seven mixed mesotheliomas. COX-2 staining was always cytoplasmic, while p53, p21 and p27 staining was preferentially nuclear although cytoplasmic positivity was occasionally seen for p2l and p27. Both cytoplasmic and nuclear stainings were considered positive. Figure 1 shows some typical immunohistochemical stainings for COX-2 (fig $1 \mathrm{~A}$ and B) and p27 (fig $1 \mathrm{C}$ and D). p53 and p21 immunostainings on the same group of mesothelioma samples have been reported elsewhere. $^{23} 24$
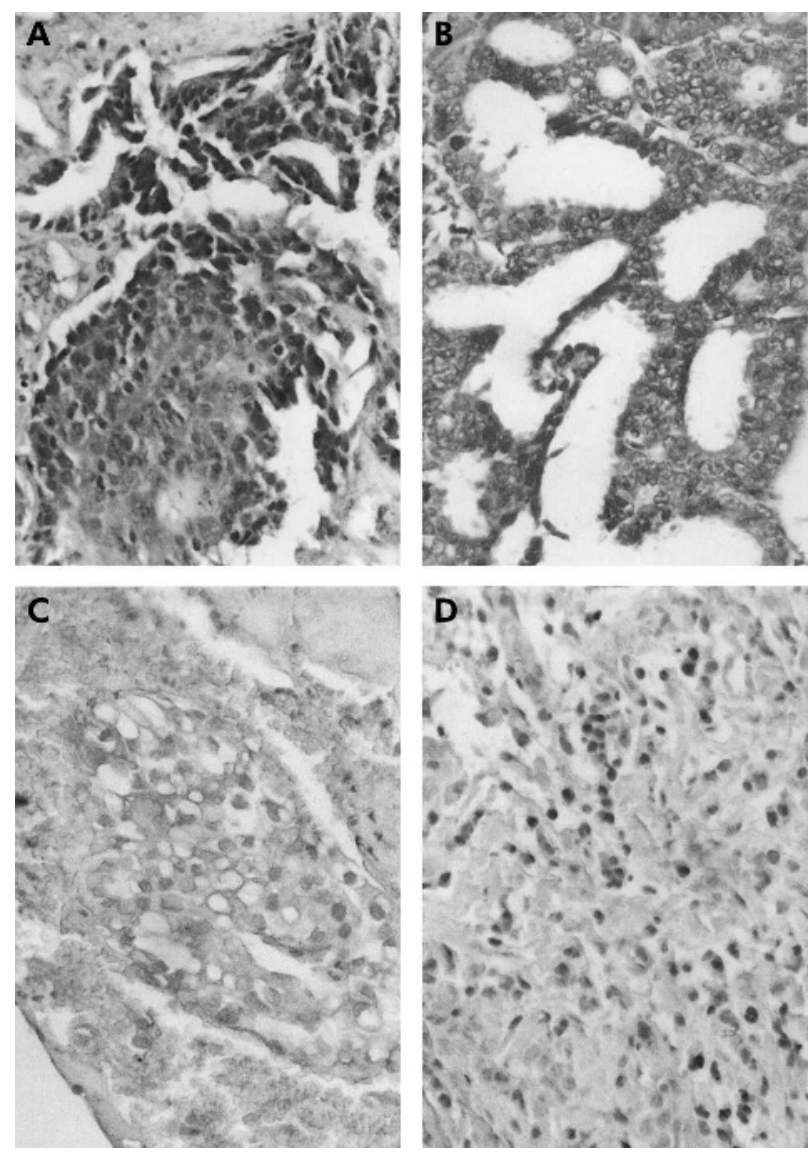

Figure 1 Immunhistochemical stainings of COX-2 (A and B) and p27 $(C$ and $D)$ in human pleural mesotheliomas (original magnification $\times 250$ ). 


\begin{tabular}{|c|c|c|c|c|c|c|}
\hline $\begin{array}{l}\text { Patient } \\
\text { no }\end{array}$ & Histology & COX-2 score* & p53 score & p21 score & p27 score $†$ & $\begin{array}{l}\text { Survival time } \\
\text { (weeks) }\end{array}$ \\
\hline 1 & Epithelial & 1 & 2 & 2 & 2 & 18 \\
\hline 2 & Epithelial & 1 & 1 & 2 & 2 & 15 \\
\hline 3 & Epithelial & 2 & $i$ & 1 & 2 & 6 \\
\hline 4 & Epithelial & 2 & 2 & $i$ & 1 & 1 \\
\hline 5 & Epithelial & 1 & 2 & 2 & 2 & 12 \\
\hline 6 & Epithelial & 2 & 1 & 2 & 2 & 10 \\
\hline 7 & Epithelial & 1 & 2 & 2 & 2 & 18 \\
\hline 8 & Epithelial & 2 & 1 & 1 & 1 & 9 \\
\hline 9 & Epithelial & 2 & 2 & 1 & 1 & 7 \\
\hline 10 & Epithelial & 1 & 1 & 1 & 2 & 14 \\
\hline 11 & Epithelial & 1 & 1 & 2 & 2 & 13 \\
\hline 12 & Epithelial & 2 & 1 & 1 & 1 & 4 \\
\hline 13 & Epithelial & 2 & 1 & $i$ & 1 & 3 \\
\hline 14 & Epithelial & 2 & $i$ & $i$ & $i$ & 5 \\
\hline 15 & Epithelial & 1 & 2 & 2 & 1 & 10 \\
\hline 16 & Epithelial & 1 & 1 & 2 & 2 & 14 \\
\hline 17 & Sarcomatoid & 1 & 2 & 2 & 2 & 9 \\
\hline 18 & Sarcomatoid & 2 & 1 & 1 & 1 & 3 \\
\hline 19 & Sarcomatoid & 2 & $i$ & 1 & 1 & 5 \\
\hline 20 & Sarcomatoid & 2 & 2 & 1 & 1 & 4 \\
\hline 21 & Sarcomatoid & 2 & 1 & 2 & 1 & 8 \\
\hline 22 & Sarcomatoid & 1 & 2 & 2 & 2 & 16 \\
\hline 23 & Mixed & 2 & 1 & 1 & 1 & 6 \\
\hline 24 & Mixed & 2 & 1 & 1 & 1 & 4 \\
\hline 25 & Mixed & 2 & 2 & $i$ & 1 & 1 \\
\hline 26 & Mixed & 2 & 2 & 2 & 2 & 11 \\
\hline 27 & Mixed & 2 & 1 & 2 & 2 & 9 \\
\hline 28 & Mixed & 2 & 1 & 2 & 2 & 15 \\
\hline 29 & Mixed & 2 & 1 & 1 & 1 & 8 \\
\hline
\end{tabular}

\section{Correlation between immunohistochemical and pathological parameters}

A positive statistically significant correlation was recorded by rank correlation matrix between p27 and p2 1 expression, and a negative correlation was found between COX-2 expression and both p27 and p21 (table 2). Interestingly, there was no statistically significant correlation between p53 and all the other immunohistochemical parameters. No correlation was seen between immunohistochemical parameters and mesothelioma histological type.

\section{Immunohistochemical and pathological parameters and patient survival}

Univariate analysis showed that overall survival was strongly influenced by p21, p27, and COX-2 expression. The median survival in patients with low p21 or p27 expression was longer than in those with high p21 or p27 expression. The median survival in patients with low COX-2 expression was 14 months compared with 5 months in those with high COX2 expression. On the other hand, p53 expression and histological type did not influence the overall survival in our patient population (table 3). Kaplan-Meier survival plots for all patients are shown in fig 2. There was a significant association between high levels of COX-2 or low levels of p27 and p21 and poor outcome $(\mathrm{p}<0.0001)$, while p53 levels did not significantly affect outcome.

Interestingly, chemotherapy and radiotherapy did not have any effect on overall survival in univariate analysis (data not shown).

Multivariate Cox regression analysis showed that the only immunohistochemical parameter to influence overall survival in patients with malignant mesothelioma was COX-2, the calculated relative risk of death in patients with low COX-2 expression being significantly lower $(0.143 ; \mathrm{p}=0.01$, table 4$)$. The other two parameters significantly associated with prognosis by univariate analysis (p27 and p21) did not influence the overall survival when evaluated by multivariate analysis, although p27 achieved borderline significance in multivariate analysis $(\mathrm{p}=0.066)$.

\section{DISCUSSION}

We have analysed the expression of COX-2, p27, p21 and p53 and correlated these data with several clinical parameters in a group of 29 mesothelioma specimens positive by polymerase

Table 2 Rank correlation matrix (and statistical significance) between molecular markers in patients with malignant mesothelioma

\begin{tabular}{llcccc}
\hline & Histology & COX-2 & p53 & p21 & p27 \\
\hline COX-2 & 0.589 & & -2.557 & -16.546 & -16.163 \\
& 0.720 & & 0.110 & $<0.0001$ & 0.001 \\
p53 & 0.736 & -2.557 & & 1.691 & 0.279 \\
& 0.792 & 0.110 & & 0.194 & 0.710 \\
p21 & 0.262 & -16.546 & 1.691 & & 16.905 \\
& 1.000 & $<0.0001$ & 0.194 & & $<0.0001$ \\
p27 & 1.073 & -16.563 & 0.279 & 17.905 & \\
& 0.693 & 0.001 & 0.710 & $<0.0001$ & \\
\hline
\end{tabular}



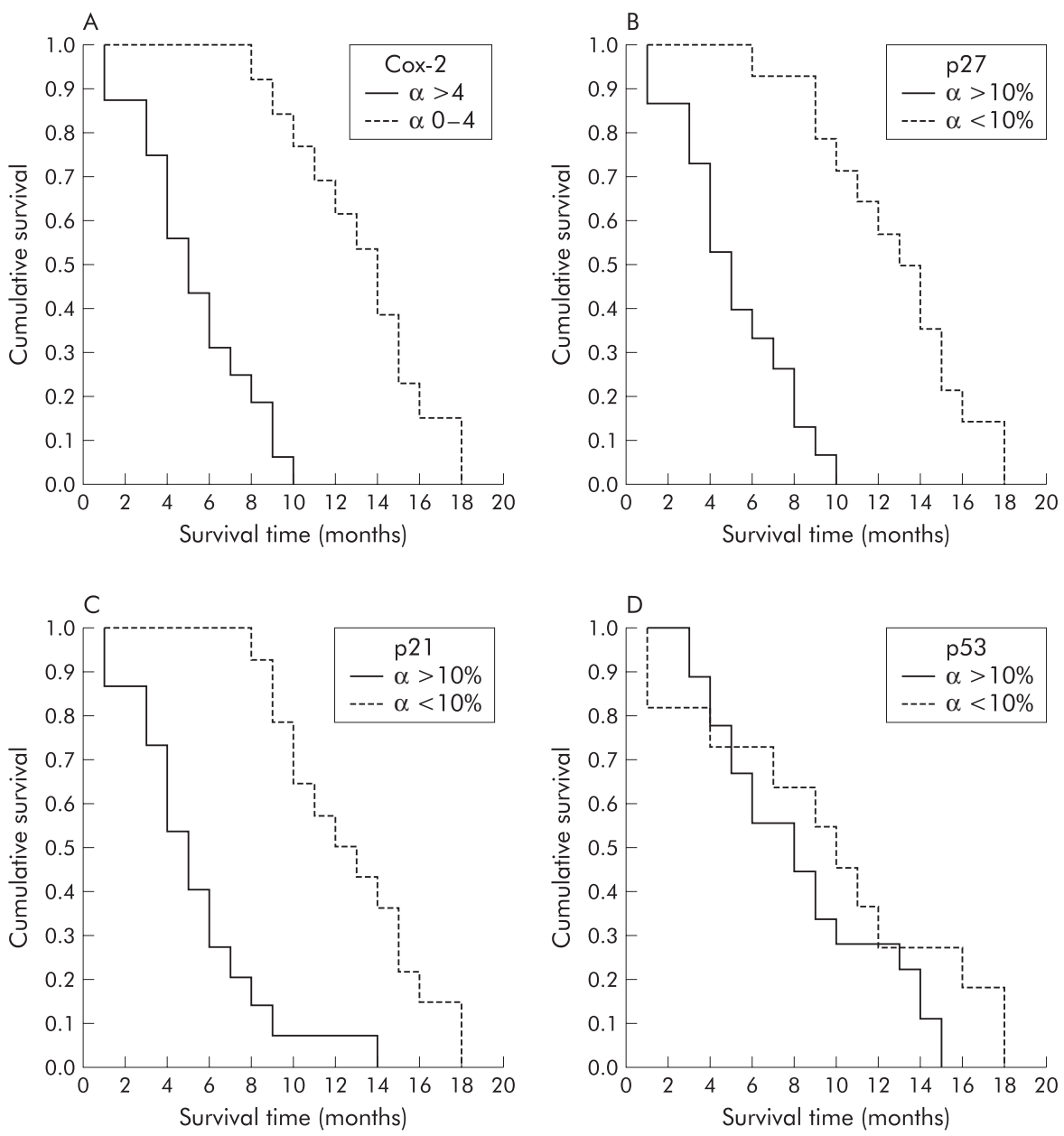

Figure 2 Kaplan-Meier survival plots for (A) COX-2, (B) p27, (C) p21, and (D) p53 levels.

chain reaction for SV40 sequences. ${ }^{25}$ We did not find any relationship between the levels of expression of the parameters analysed and the histological types in these patients. These data agree with the lack of correlation reported by others between COX-2, p21, and p53 and type of mesothelioma, ${ }^{1723}$ but other groups have found a significant

Table 3 Univariate analysis of survival (in weeks) and pathological and immunohistochemical parameters in patients with malignant mesothelioma

\begin{tabular}{lcc}
\hline & Median $(95 \% \mathrm{Cl})$ survival & p value \\
\hline Histology & $10.00(8.06$ to 11.94$)$ & 0.4927 (NS) \\
$\quad$ Epithelial & $5.00(1.20$ to 9.80$)$ & \\
$\quad$ Sarcomatoid & $8.00(3.87$ to 13.13$)$ & \\
$\quad \begin{array}{l}\text { Mixed } \\
\text { COX-2 }\end{array}$ & $14.00(11.71$ to 16.29$)$ & $<0.0001$ \\
$0-4$ & $5.00(3.06$ to 6.94$)$ & \\
$>4$ & & \\
p53 & $8.00(3.87$ to 12.13$)$ & 0.1977 (NS) \\
$<10 \%$ & $10.00(5.68$ to 14.32$)$ & \\
$>10 \%$ & $5.00(3.47$ to 6.54$)$ & $<0.0001$ \\
p21 & $12.00(8.33$ to 15.67$)$ & \\
$<10 \%$ & & \\
$>10 \%$ & $5.00(3.51$ to 6.49$)$ & $<0.0001$ \\
p27 & $13.00(10.56$ to 15.44$)$ & \\
$<10 \%$ & &
\end{tabular}

correlation between p27 expression and epithelioid histotype. $^{2021}$

We found that a high level of COX-2 expression and low p21 and p27 expression were associated with a statistically significant decrease in survival. In multivariate analysis, COX-2 expression predicted outcome independently of all other variables. These findings indicate that high COX-2 expression in tumour cells is associated with clinically more aggressive mesotheliomas and is a strong predictor of poor survival.

Recent studies have underlined the involvement of COX-2 in the natural history of mesothelioma and have also established its prognostic value. ${ }^{17}{ }^{18}$ However, p21 and p27 have also been shown to have a significant role in the

Table 4 Multivariate Cox regression analysis of overall survival in patients with malignant mesothelioma

\begin{tabular}{lll}
\hline & Hazard ratio $(95 \% \mathrm{Cl})$ & p value \\
\hline $\begin{array}{l}\text { COX-2 } \\
0-4\end{array}$ & $0.143(0.032$ to 0.646$)$ & 0.011 \\
$>4$ & 1 & \\
$\mathrm{p} 21$ & & \\
$<10 \%$ & $2.191(0.685$ to 7.005$)$ & 0.186 (NS) \\
$>10 \%$ & 1 & \\
$\mathrm{p} 27$ & & \\
$<10 \%$ & $4.016(0.913$ to 17.653$)$ & 0.066 (NS) \\
$>10 \%$ & 1 & \\
\hline
\end{tabular}


progression of human mesothelioma. ${ }^{20-22}$ Interestingly, while p53 overexpression is a common event in mesothelioma, ${ }^{24}$ it is rarely mutated or inactivated..$^{29}$

The data presented here showing COX-2 expression as the strongest predictor of a poor outcome not only confirm previous published results but also shed new light on the role of COX-2 in the pathogenesis and progression of mesothelioma. In vitro studies on mesangial cells have recently shown that COX-2 exerts its inhibition on cell proliferation by a mechanism that is independent of prostaglandin synthesis but involves p53, p21, and p27. ${ }^{19}$ Based on our immunohistochemical data, it is possible to hypothesise a model where the weaker the COX-2 mediated induction of p21 and p27, the more aggressive is the tumour and the poorer the prognosis.

Based on this model, we would expect to find that p53 behaves in a similar way to p21 and p27, but our data do not show any correlation between p53 and COX-2 expression. This can be explained by the fact that all the mesothelioma specimens analysed were positive for the presence of SV40like sequences. ${ }^{25}$ Consistent with the possible role of SV40 oncoproteins in the pathogenesis of mesothelioma, ${ }^{4}$ it has been observed that mutations involving p53 are extremely rare in these neoplasms ${ }^{29}$ and that SV40-Tag isolated from mesotheliomas is able to bind to p53 and to the RB family proteins. ${ }^{25}{ }^{30}$ Inactivation of p53 mediated by SV40 could at least partly account for this phenomenon, even if the relevance of SV40 in the pathogenesis of mesothelioma is not universally accepted. ${ }^{31}$ Another possible explanation could be derived from the observation that the activity of the COX-2 inhibitor NS398 is not affected by the antiproliferative effects of p53 in human mesothelioma cell lines, ${ }^{17}$ thus showing the lack of influence of p53 on the COX-2 pathway. However, the role of p53 in mesothelioma still remains unclear.

It is not possible to exclude the possibility that molecular factors other than p21 and p27 might contribute to the complex COX-2 mediated inhibition of proliferation. In fact, the combined action of a number of factors is necessary to induce COX-2 dependent cell cycle arrest. Nevertheless, the tumorigenic effects of COX-2 could be divided into two distinct types: a direct effect on tumour cells and an effect on non-tumour cells such as tumour-nurturing blood vessels and immune competent cells. ${ }^{32}$ Our finding that only COX-2 expression was significantly associated with survival in multivariate analysis further confirms this hypothesis.

One important limitation of this study is the absence of data on recognised clinical variables associated with prognosis such as performance status and the fact that it did not detect an association between histological type and prognosis. Moreover, the data are retrospective and essentially observational in nature and cannot therefore explain the functional mechanisms by which COX-2 promotes tumour growth. Further studies on larger independent groups of patients are needed to elucidate the interactions between oncogenes, tumour suppressor genes, and COX-2 expression in mesotheliomas.

In conclusion, this is the first study of which we are aware of the relationship between COX-2, p21, p27 and survival in patients with mesothelioma. The data suggest that COX-2 expression may be a useful prognostic parameter and thus support further investigations into the clinical usefulness of COX-2 inhibitors in the treatment of malignant mesotheliomas.

\section{Authors' affiliations}

A Baldi, M Pia Di Marino, F Baldi, Department of Biochemistry and Biophysics, "F Cedrangolo", Section of Anatomic Pathology, Second University of Naples, Naples, Italy
D Santini, B Vincenzi, G Tonini, Section of Oncology, "Campus BioMedico" University, Rome, Italy

F Vasaturo, M Piccoli, S Scarpa, Department of Experimental Medicine and Pathology, "La Sapienza" University, Rome, Italy

M Santini, G Vicidomini, Department of Thoracic Surgery, Second University of Naples, Naples, Italy

V Esposito, G Liuzzi, Department of Cardiological, Respiratory and Thoracic Medical Sciences, Second University of Naples, and Third Division of Infective Diseases, D Cotugno Hospital, Naples, Italy

A M Groeger, Department of Cardio-Thoracic Surgery, University of

Vienna, Austria

V Esposito, A M Groeger, International Society for the Study of Comparative Oncology (ISSCO), Silver Spring, MD, USA

Funding: International Society for the Study of Comparative Oncology Inc (ISSCO, President H E Kaiser) Silver Spring, MD, USA; FUTURAOnlus; General Broker Service; MIUR and Second University of Naples.

\section{REFERENCES}

1 Peto J, DeCarli A, La Vecchia C, et al. The European mesothelioma epidemic. Br J Cancer 1999;79:666-72

2 Attanos RI, Gibbs AR. Pathology of malignant mesothelioma. Histopathology 1997:30:403-18.

3 Hughes JM, Weill H. Asbestos exposure: quantitative assessment of risk. Am Rev Respir Dis 1986;133:5-13.

4 Carbone M, Fisher S, Powers A, et al. New molecular and epidemiological issues in mesothelioma: role of SV40. J Cell Physiol 1999; 180:167-72.

5 Sugarbaker DJ, Flores RM, Jaklitsch MT, et al. Resection margins, extrapleural nodal status, and cell type determine postoperative longterm survival in trimodality therapy of malignant pleural mesothelioma: results in 183 patients. J Thorac Cardiovasc Surg 1999; 117:54-63.

6 Pass HI, Robinson BW, Testa JR, et al. Emerging translational therapies for mesothelioma. Chest 1999;116:455-60S.

7 Rusch VW, Venkatraman ES. Important prognostic factors in patients with malignant pleural mesothelioma, managed surgically. Ann Thorac Surg 1999;68: 1799-804.

8 Johansson L, Linden CJ. Aspects of histopathologic subtype as a prognostic factor in 85 pleural mesotheliomas. Chest 1996;109:109-14.

9 Beer TW, Buchanan R, Matthews AW, et al. Prognosis in malignant mesothelioma related to MIB 1 proliferation index and histological subtype. Hum Pathol 1998;29:249-51.

10 Uefuji K, Ichikura T, Mochizuki H. Cycloxygenase-2 expression is related to prostaglandin biosynthesis and angiogenesis in human gastric cancer. Clin Cancer Res 2000;6:135-8.

11 Grosch S, Tegeder I, Niederberger E, et al. COX-2 independent induction of cell cycle arrest and apoptosis in colon cancer cells by the selective COX-2 inhibitor celecoxib. FASEB J 2001;15:2742-4.

12 Tsuji S, Tsujii M, Kawano S, et al. Cyclooxygenase-2 upregulation as a perigenetic change in carcinogenesis. J Exp Clin Cancer Res 2001;20:117-29.

13 Sheehan KM, Sheahan K, O'Donoghue DP, et al. The relationship between cyclooxygenase- 2 expression and colorectal cancer. JAMA 1999;282:1254-7.

14 Hida T, Yatabe Y, Achiwa H, et al. Increased expression of cyclooxygenase-2 occurs frequently in human lung cancers, specifically in adenocarcinomas. Cancer Res 1998;58:3761-4.

15 Murata H, Kawano S, Tsuji S, et al. Cyclooxygenase-2 overexpression enhances lymphatic and metastasis in human gastric carcinoma. Am J Gastroenterol 1999;94:451-5.

16 Baer AN, Green FA. Cyclooxygenase activity of cultured human mesothelial cells. Prostaglandins 1993;46:37-49.

17 Marrogi A, Pass HI, Khan M, et al. Human mesothelioma samples overexpress both cyclooxygenase-2 (COX-2) and inducible nitric oxide synthase (NOS2): in vitro antiproliferative effects of a COX-2 inhibitor. Cancer Res 2000;60:3696-700.

18 Edwards JG, Faux SP, Plummer SM, et al. Cyclooxygenase-2 expression is a novel prognostic factor in malignant mesothelioma. Clin Cancer Res 2002:8:1857-62.

19 Zahner G, Wolf G, Ayoub M, et al. Cyclooxygenase-2 overexpression inhibits platelet-derived growth factor-induced mesangial cell proliferation through induction of the tumour suppressor gene p53 and the cyclin-

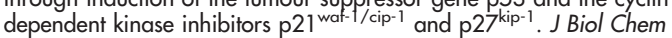
2002;277:9763-71.

20 Bongiovanni $M$, Cassoni P, De Giuli P, et al. p2 $7^{\text {kip } 1}$ immunoreactivity correlates with long-term survival in pleural malignant mesothelioma. Cancer 2001;92:1245-50.

21 Beer TW, Shepherd P, Pullinger NC. p27 immunostaining is related to prognosis in malignant mesothelioma. Histopathology 2001;38:535-41.

22 Isik R, Metintas M, Gibbs AR, et al. p53, p21 and metallothionein immunoreactivities in patients with malignant pleural mesothelioma: correlations with environmental asbestos exposure. Respir Med 2001;95:588-93.

23 Baldi A, Groeger AM, Esposito V, et al. Expression of p21 in SV40 large T antigen positive human pleural mesothelioma: relationship with survival. Thorax 2002;57:353-6. 
24 Esposito V, Baldi A, De Luca A, et al. p53 immunostaining in differential diagnosis of pleural mesothelial proliferations. Anticancer Res 1997;17:733-6.

25 De Luca A, Baldi A, Esposito V, et al. The retinoblastoma gene family pRb/ p105, p107, pRb2/p130 and simian virus-40 large T-antigen in human mesotheliomas. Nature Med 1997;3:913-6.

26 Kaplan EL, Meier P. Nonparametric estimation from incomplete observations. $J$ Am Stat Assoc 1958;53:457-81.

27 Peto R, Pike MC, Armitage $P$, et al. Design and analysis of randomised clinical trials requiring prolonged observation of each patient. $\mathrm{Br} J$ Cancer 1977;35:1-39.
28 Cox DR. Regression models and life tables. J R Stat Soc 1972;34:187-220

29 Mor O, Yaron P, Huszar M, et al. Absence of p53 mutations in malignant mesotheliomas. Am J Respir Cell Mol Biol 1997;16:9-13.

30 Carbone M, Rizzo P, Grimley PM, et al. Simian virus- 40 large-T antigen binds p53 in human mesotheliomas. Nature Med 1997;3:908-12.

31 Mulatero C, Surentheran T, Breuer J, et al. Simian virus 40 and human pleural mesothelioma. Thorax 1999;54:60-1.

32 Subbaramaiah K, Altorki N, Chung WJ, et al. Inhibition of cyclooxygenase-2 gene expression by p53. J Biol Chem 1999;274:10911-5.

\section{LUNG ALERT}

Procalcitonin may guide antibiotic treatment for LRTI

$\Delta$ Christ-Crain M, Jaccard-Stoiz D, Bingisser R, et al. Effect of procalcitonin-guided treatment on antibiotic use and outcome in lower respiratory tract infections: cluster-randomised, single-blinded intervention trial. Lancet 2004;363:600-7

$\mathrm{t}$ is often difficult to decide which patients with suspected lower respiratory tract infection (LRTI) should be prescribed antibiotics. Circulating calcitonin precursors are higher in severe bacterial infections than in other illnesses. In this prospective single blind study 243 patients with suspected LRTI were randomised to a standard care group $(\mathrm{n}=119)$ treated with antibiotics on the basis of clinical findings and routine investigations, or a procalcitonin group $(\mathrm{n}=124)$ guided by a new procalcitonin assay with a sensitivity of $0.06 \mu \mathrm{g} / \mathrm{l}$. In this group, antibiotics were strongly discouraged if procalcitonin levels were $<0.10 \mu \mathrm{g} / \mathrm{l}$, discouraged if levels were $\geqslant 0.10-0.25 \mu \mathrm{g} / \mathrm{l}$, advised if levels were $\geqslant 0.25-0.50 \mu \mathrm{g} / \mathrm{l}$, and strongly recommended if levels were $\geqslant 0.50 \mu \mathrm{g} / \mathrm{l}$, with re-evaluation after 6-24 hours in both groups.

The primary end point was the use of antibiotics: 99 (83\%) subjects in the standard care group and $55(44 \%)$ in the procalcitonin group received antibiotic treatment. The adjusted relative risk of antibiotic exposure in the procalcitonin group was 0.49 (95\% CI 0.44 to 0.55 ; $\mathrm{p}<0.0001)$ compared with the standard group. The reduction in antibiotic use was $47 \%$ in LRTI and $56 \%$ in acute exacerbations of COPD. There was no difference in laboratory and clinical outcomes including mortality, frequency and length of admission, need for ITU care, and rates of re-exacerbation of COPD and readmission after a mean (SD) of 13.0 (5.4) days. Re-evaluation after a mean (SD) of 5.3 (1.1) months showed no difference.

The fact that the study was single blind was a source of possible bias. There is no equivalent study using CRP as an alternative test. However, this study may have important clinical and economic implications.

P De

Staff Grade Physician, Respiratory Medicine, Darlington Memorial Hospital, UK; Pradip.de@btopenworld.com 